\title{
A New Intelligent Motion Planning for Mobile Robot Navigation using Multiple Adaptive Neuro-Fuzzy Inference System
}

\author{
Prases K. Mohanty* and Dayal R. Parhi \\ Robotics Laboratory, National Institute of Technology, Department of Mechanical Engineering, Rourkela, Odisha,769008, India
}

Received: 23 Sep. 2013, Revised: 21 Dec. 2013, Accepted: 22 Dec. 2013

Published online: 1 Sep. 2014

\begin{abstract}
Nowadays intelligent tools such as fuzzy inference system (FIS), artificial neural network (ANN) and adaptive neuro-fuzzy inference system (ANFIS) are mainly considered as effective and suitable methods for modeling an engineering system. This paper presents a new hybrid technique based on the combination of fuzzy inference system and artificial neural network for addressing navigational problem of autonomous mobile robot. First we developed an adaptive fuzzy controller with four input parameters, two output parameters and three parameters each. Afterwards each adaptive fuzzy controller acts as a single takagi-sugeno type fuzzy inference system, where inputs are front obstacle distance (FOD), left obstacle distance (LOD), right obstacle distance (ROD) (from robot), heading angle (HA) (angle to target) and output corresponds to the wheel velocities ( Left wheel and right wheel) for the mobile robot. The effectiveness, feasibility and robustness of the proposed navigational controller have been demonstrated by means of simulation experiments. The real time experimental results were verified with simulation experiments, showing that the proposed navigational algorithm consistently performs better results to navigate the mobile robot safely in a completely or partially unknown environment.
\end{abstract}

Keywords: ANFIS, Mobile robot, Navigation, Obstacle avoidance

\section{Introduction}

Autonomous mobile robots have generated much interest in recent years due to their ability to perform relatively challenging tasks in hazardous or remote environments. At present mobile robots have been effectively used in various areas of engineering such as aerospace research, nuclear research, production engineering etc. The major objective in the current robotic research area is to find a collision free path from a given start position to predefined target point. In general path planning methods are classified as local and global depending upon the surrounding environment. In global path planning the surrounding environment is completely known to the mobile robot so the path travelled by the mobile robot is predefined, where as in local path planning the environment is completely unknown or partially known to the mobile robot. So various sensors are used to perceive the information about the surrounding environment and plan the motion accordingly. Many exertions have been paid in the past to improve various robot navigation techniques.

In literature review, there can be found several researchers have been addressed on many intelligent techniques for path planning of mobile robot. Many authors have considered a controller with complete information of the environment [1,2]. Due to the complexity and uncertainty of the path planning problem, classical path planning methods, such as visibility graph [3], voronoi diagrams [4], grids [5], cell decomposition [6], artificial potential field [7], rule based methods [8], and rules learning techniques [9] are not appropriate for path planning in dynamic environments. The use of the above algorithms for path finding for mobile robot requires more time and the finding of this path will not completely feasible for real-time movement. There are many fuzzy logic techniques using various implementations or in combination with other techniques [10-14]. Mobile robot path planning based on neural network approaches presented by many

\footnotetext{
*Corresponding author e-mail: pkmohanty30@gmail.com
} 
researchers [15-18]. Among the intelligent techniques ANFIS is a hybrid model which combines the adaptability capability of artificial neural network and knowledge representation of fuzzy inference system [19]. Song and Sheen [20] developed a pattern recognition method based on fuzzy-neuro network for reactive navigation of a car-like robot. Li et al. [21]suggested a neuro-fuzzy technique for behavior based control of a car-like robot that navigates among static obstacles. Navigation of multiple mobile robots using neuro-fuzzy technique addressed by Pradhan et al. [22]. In this design controller, output from the neural network given as input to the fuzzy controller to navigate the mobile robot successfully in the clutter environment. Experimental verification also has been done with the simulation result to prove the validity of the developed technique. Navigation of mobile robots using adaptive neural-fuzzy system discussed by Nefti et al. [23]. Different sensor based information they have given to the SugenoTakagi fuzzy controller and output from the controller is the robot orientation. Experimental results settle the importance of the methodology when dealing with navigation of a mobile robot in a completely or partially unknown environment. A neuro-fuzzy controller based mobile robot navigation presented by Kim and Trivedi [24]. In this study they have implemented neural integrated fuzzy controller to control the mobile robot motion in terms of steering angle, heading direction, and speed. Control of mobile robot based on neuro-fuzzy technique discussed by Godjevac and Steele [25]. In this paper they have shown how neuro-fuzzy controller can be achieved using a controller based on the Takagi-Sugeno design and a radial basis function neural network for its implementation. To determine collision-free path of mobile robot navigating in a dynamic environment using neuro-fuzzy technique presented by Hui et al. [26]. The performances of neuro-fuzzy approaches are compared with other approaches (GA, Mamdani) and it is found that neuro-fuzzy approaches are found to perform better than the other approaches.

In this paper we propose a new intelligent navigational controller for solving navigation problem for mobile robot in a completely or partially unknown environment. A new MANFIS (Fig. 1) (Multiple Adaptive Neuro-Fuzzy Inference System) motion controller has been developed to solve the optimization problem. Finally simulation results using MATLAB are presented to verify the effectiveness of the proposed path planner in various scenarios populated by stationary obstacles.

\section{Kinematic Analysis of Mobile Robot}

To control the movement of a mobile robot we need Kinematic analysis of the robot. The kinematics analysis of differentially steered wheeled mobile robots in a two-dimensional plane can be done in one of two ways: either by Cartesian or polar coordinates. It is assumed that

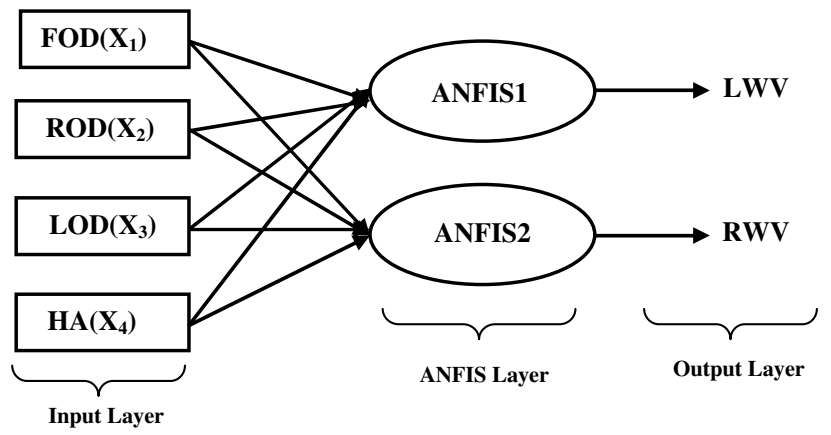

Fig. 1: Proposed MANFIS (Multiple ANFIS) controller for Mobile Robot Navigation

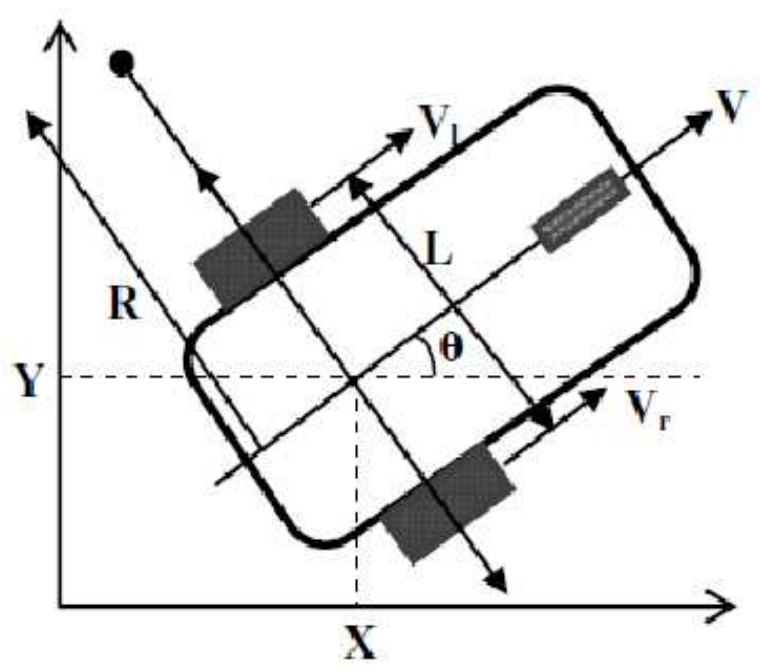

Fig. 2: Mobile Robot Kinematic Parameters

the mobile robot moves without slipping on a plane, that means there is a pure rolling contact between the wheels and the ground and also there is no lateral slip between the wheel and the plane. The modeling in Cartesian coordinates is the most common use and the discussion will be limited to modeling in Cartesian coordinates. The robot has two fixed standard wheels and one caster wheel and is differentially driven by skid steer motion. The two driving wheels are independently driven by two motors to acquire the motion and orientation. Both the wheels have same diameter $2 \mathrm{r}$ (Fig. 2). The driving wheels are separated by distance L. The position of the robot in the 2-D plane at any instant is defined by the situation in Cartesian coordinates and the heading with respect to a global frame of reference.

$V_{r}(t)=$ Linear velocity of right wheel

$V_{l}(t)=$ Linear velocity of left wheel

$\mathrm{r}=$ Nominal radius of each wheel

$\omega(t)=$ Angular velocity of the wheel 
$\mathrm{R}=$ Instantaneous curvature radius of the robot trajectory, relative to the mid-point axis

$\mathrm{R}-\mathrm{L} / 2=$ Curvature radius of trajectory described by left wheel

$\mathrm{R}-\mathrm{L} / 2=$ Curvature radius of trajectory described by right wheel

$$
V(t)=\omega(t) R=\frac{1}{2}\left(V_{r}(t)+V_{l}(t)\right)
$$

So the tangential velocity in the global reference plane

$$
\begin{gathered}
\dot{x}(t)=V(t) \cos \theta(t) \\
\dot{y}(t)=V(t) \sin \theta(t) \\
\dot{\theta}(t)=\omega(t)
\end{gathered}
$$

\section{Architecture of Multiple Adaptive Neuro-Fuzzy Inference System (MANFIS) for Current Analysis}

Adaptive network-based fuzzy inference system (ANFIS) is one of hybrid intelligent neuro-fuzzy structure and it functioning under Takagi-Sugeno-type fuzzy interference system, which was designed by Jang [19] in 1993.

There are two learning paradigms are used in ANFIS to show the mapping between input and output data and to compute optimized of fuzzy membership functions. These learning methods are back propagation and hybrid. Parameters associated with fuzzy membership functions will modify through the learning process.

As for the prediction of left wheel velocity (LWV) and right wheel velocity (RWV) for mobile robot we assume each adaptive neuro-fuzzy controller under consideration of four inputs parameters i.e. Front obstacle distance(FOD) ( $\left.x_{1}\right)$, Right obstacle distance(ROD) $\left(x_{2}\right)$, Left obstacle distance(LOD) $\left(x_{3}\right)$, Heading angle(HA) $\left(x_{4}\right)$ and each input variable has three bell membership functions(MF) (Fig.4) $A_{1}$ (Near), $A_{2}$ (Medium) and $A_{3}$ (Far), $B_{1}$ (Near), $B_{2}$ (Medium) and $B_{3}$ (Far), $C_{1}$ (Near), $C_{2}$ (Medium) and $C_{3}$ (Far), $D_{1}$ (Negative), $D_{2}$ (Zero) and $D_{3}$ (positive) respectively, then a Takagi-Sugeno-type fuzzy inference system if-then rules are defined as follows;

\section{THEN}

Rule: $\operatorname{IF}\left(x_{1}\right.$ is $A_{i}$ and $x_{2}$ is $B_{i}$ and $x_{3}$ is $C_{i}$ and $x_{4}$ is $\left.D_{i}\right)$

$f_{n}($ wheel velocity $)=p_{n} x_{1}+q_{n} x_{2}+r_{n} x_{3}+s_{n} x_{4}+u_{n}$

A, B, C, and D are the fuzzy membership sets for the input variables $x_{1}, x_{2}, x_{3}$ and $x_{4}$ respectively. where, $\mathrm{i}=1,2,3$ and $p_{n}, q_{n}, r_{n}, s_{n}$ and $u_{n}$ are the linear parameters of function $f_{n}$ and changing these parameters we can modify the output of ANFIS controller.

The function of each layer in ANFIS structure (Fig. 5) is discussed as follows:

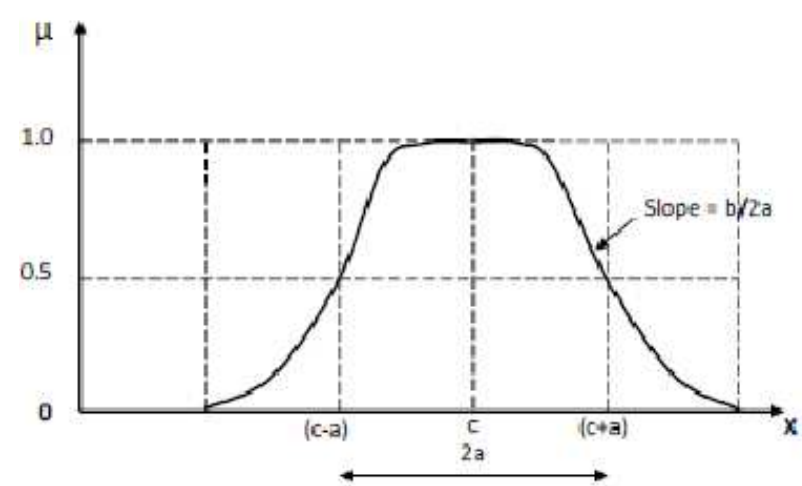

Fig. 3: Parameters in the bell membership function

Input Layer: In this layer nodes receive signals from array of sensors $\left(x_{1}, x_{2}, x_{3}\right.$ and $\left.x_{4}\right)$ which specify the position of the obstacles and target. That is defined as

$$
\left.\begin{array}{r}
O_{0, F O D}=X_{1}, \\
O_{0, R O D}=X_{2}, \\
O_{0, L O D}=X_{3}, \\
O_{0, H A}=X_{4}
\end{array}\right\}
$$

First Layer: This layer is the adaptive fuzzy layer. Neurons in this layer complete the fuzzification process. Every node in this stage is an adaptive node (square node) and calculating the membership function value in fuzzy set. For four inputs the outputs from nodes in this layer are presented as

$$
\left.\begin{array}{rl}
O_{1, i} & =\mu_{A_{i}}\left(X_{1}\right), \\
O_{1, i} & =\mu_{B_{i}}\left(X_{2}\right), \\
O_{1, i} & =\mu_{C_{i}}\left(X_{3}\right), \\
O_{1, i} & =\mu_{D_{i}}\left(X_{4}\right)
\end{array}\right\}
$$

Here $O_{1}, \mathrm{i}$ is the bell shape membership grade of a fuzzy set $\mathrm{S}\left(A_{i}, B_{i}, C_{i}\right.$ and $\left.D_{i}\right)$ and it specifying the degree to which the given inputs $\left(x_{1}, x_{2}, x_{3}\right.$ and $\left.x_{4}\right)$ satisfies the quantifier S. Membership functions for A, B, $\mathrm{C}$ and $\mathrm{D}$ considered are the bell shape function and defined as follows;

$$
\begin{aligned}
\mu_{A_{i}}(x) & =\frac{1}{1+\left[\left(\frac{x_{1}-c_{i}}{a_{i}}\right)^{2}\right]^{b_{i}}}, \\
\mu_{B_{i}}(x) & =\frac{1}{1+\left[\left(\frac{x_{2}-c_{i}}{a_{i}}\right)^{2}\right]^{b_{i}}} \\
\mu_{C_{i}}(x) & =\frac{1}{1+\left[\left(\frac{x_{3}-c_{i}}{a_{i}}\right)^{2}\right]^{b_{i}}} \\
\mu_{D_{i}}(x) & =\frac{1}{1+\left[\left(\frac{x_{4}-c_{i}}{a_{i}}\right)^{2}\right]^{b_{i}}}
\end{aligned}
$$


$a_{i}, b_{i}$ and $c_{i}$ (Fig. 3) are parameters that control the Centre, width and slope of the Bell-shaped function of node i respectively. Changing these parameters will give the various contour of bell shaped function as required in accordance with the data set for the problem defined. These are also known as premise parameters.

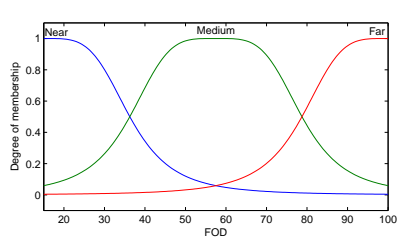

(a)

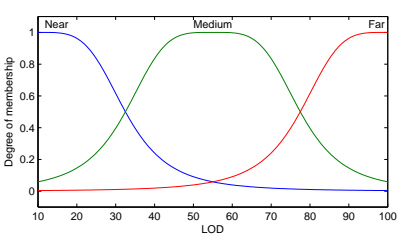

(c)

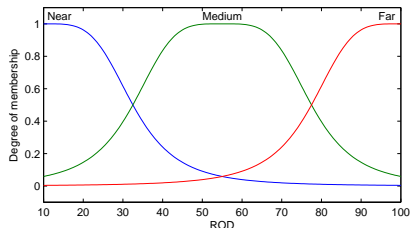

(b)

(d)

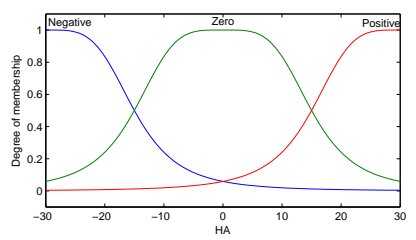

Fig. 4: (a-d) Membership functions for input parameters (FOD, ROD, LOD, HA) of MANFIS controller

Second Layer: It is also known as rule layer. Every node in this layer is a fixed node (circular) and labeled as $\pi_{n}$. Every node in this stage corresponds to a single Sugeno-Takagi fuzzy rule. Each rule point receives inputs from the respective points of layer- 2 and calculates the firing strength of the each fuzzy rule. Output from each node is the product of all incoming signals.

$$
O_{2, n}=W_{n}=\mu_{A_{i}}\left(x_{1}\right) \cdot \mu_{B_{i}}\left(x_{2}\right) \cdot \mu_{C_{i}}\left(x_{3}\right) \cdot \mu_{D_{i}}\left(x_{4}\right)
$$

There $W_{n}$ represents the firing strength or the truth value, of nth rule and $n=1,2,3, \ldots 81$ is the number of Sugeno-Takagi fuzzy rules.

Third Layer: It is the normalization layer. Every node in this layer is a fixed node (circular) and labeled as $N_{n}$. Each point in this layer receives inputs from all points in the adaptive fuzzy rule layer and calculates the normalized firing strength of a given rule. The normalized firing strength of the nth point of the nth rules firing strength to sum of all ruless firing strength.

$$
O_{3, n}=\bar{W}_{n}=\frac{W_{n}}{\sum_{n=1}^{81} W_{n}}
$$

Fourth layer: Every node in this layer is an adaptive node (square node). Each node in this layer is connected to the corresponding normalization node and also receives

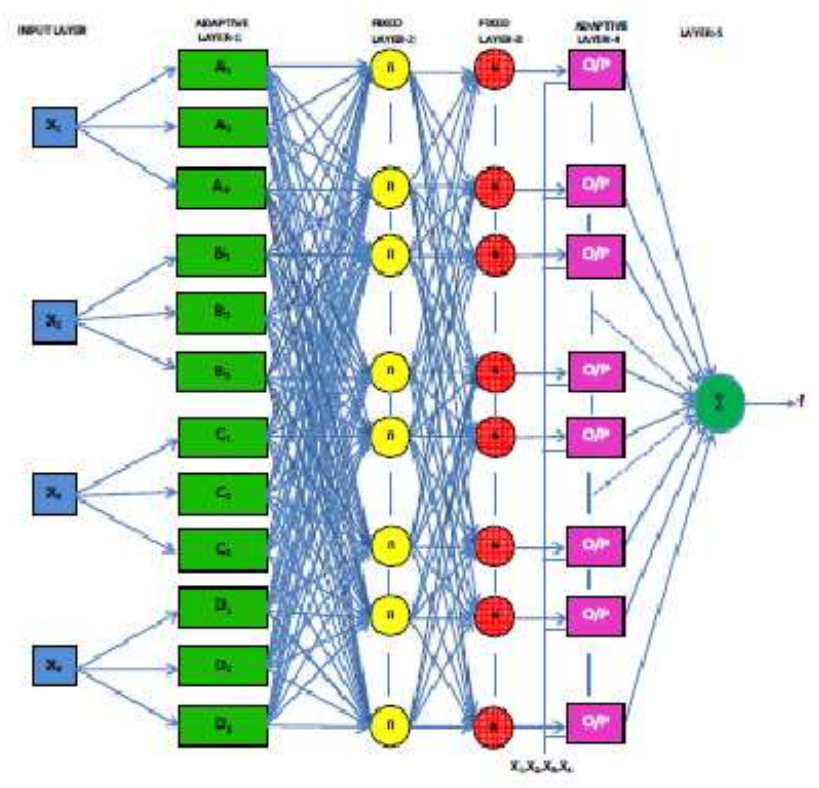

Fig. 5: The structure of ANFIS 1 network for Mobile Robot Navigation

initial inputs $x_{1}, x_{2}, x_{3}$ and $x_{4}$. A defuzzification node determines the weighted consequent value of a given rule presented as,

$O_{4, n}=\bar{W}_{n} f_{n}=\bar{W}_{n}\left[p_{n}\left(x_{1}\right)+q_{n}\left(x_{2}\right)+r_{n}\left(x_{3}\right)+s_{n}\left(x_{4}\right)+u_{n}\right]$

Where $\bar{W}_{n}$ is a normalized firing strength from layer-3 and $p_{n}, q_{n}, r_{n}, s_{n}$ and $u_{n}$ are the parameters set of this node. These parameters are also called consequent parameters.

Fifth layer: It is represented by a single summation node (circular node). This single point is a fixed point and labeled as $\sum$. This point determines the sum of outputs of all defuzzification points and gives the overall model output that is wheel velocity.

$$
O_{5, n}=\sum_{n=1}^{81} \bar{W}_{n} f_{n}=\frac{\sum_{n=1}^{81} W_{n} f_{n}}{\sum_{n=1}^{81} W_{n}}
$$

\section{Simulation experiments and discussion}

A variety of situations and routes were simulated on a computer using MATLAB version R2008a [29]. The coordinates of the sides of the paths as well as coordinates of any static obstacles were known to the MANFIS controller. Knowing the coordinates of the robot, the current navigational controller can thus calculate the distances and heading angle of the robot, as if it was sensor. In current navigation model, we developed two main reactive behaviors: one to reach the 


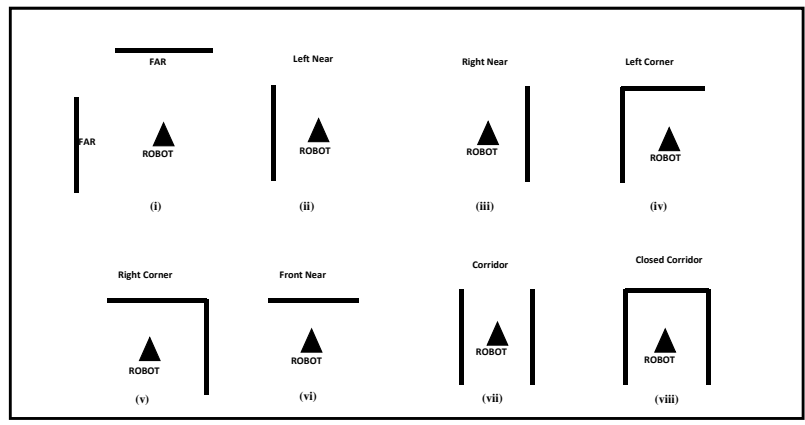

Fig. 6: Examples of various reactive behaviors

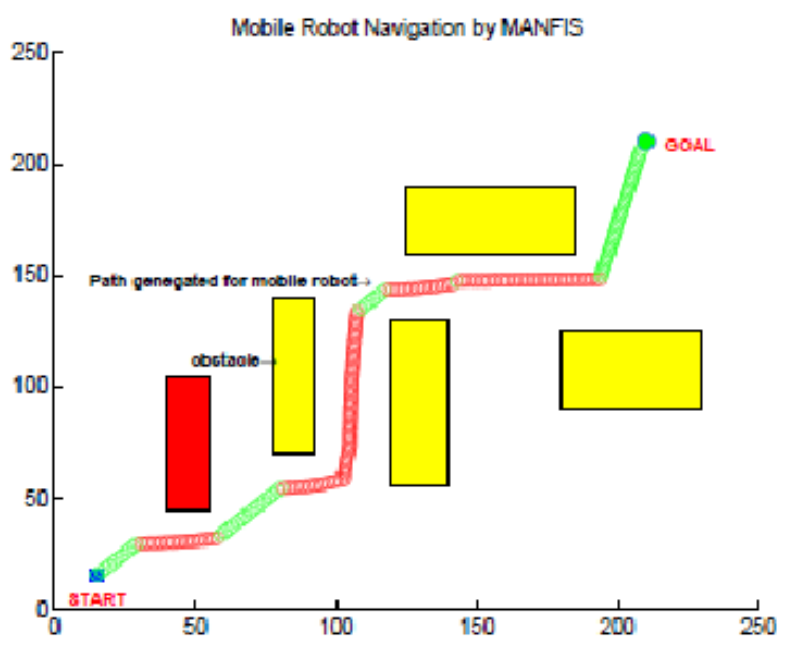

Fig. 7: Navigation of single robot using current analysis

target and the other avoiding obstacles. Fig. 6 shows the examples of different reactive behaviors.

Let us consider, every obstacle is far away from the robot and then only the reach target behavior will be actived. Other side when a robot close to an obstacle, it must changes its velocity to avoid the obstacle present on the path. Various reactive behaviors will be activated depending upon the situation between robot and obstacle. The simulation experiments (Fig.7 and Fig.8) were performed by placing the obstacles at random positions and a random heading angle to verify the various reactive behaviors developed by current navigational controller. In simulation graph red color path shows the activation of reactive behaviors by mobile robot.

\section{Comparison with other algorithms}

In this section the methods proposed in the literature survey for overcoming the local minimum problem are discussed and compared with the current approach.

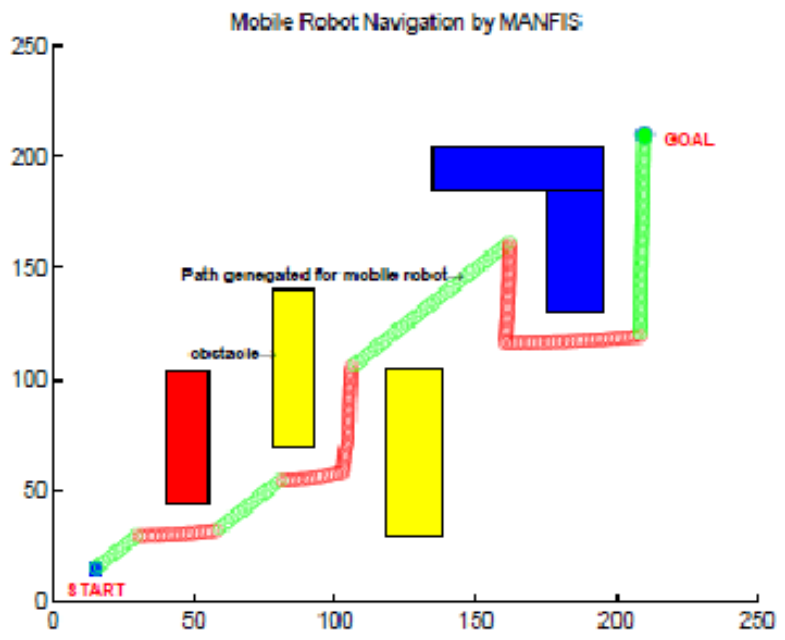

Fig. 8: Single robot escaping from corner end

1.Path planning of a wheeled mobile robot using artificial neural network (ANN) suggested by Engedy et al. [27]. In this work they have presented a neural controller with back propagation technique, which uses potential field for obstacle avoidance and the neural controller is aware of its distance sensor readings and its relative position from the target. The simulation result for the above controller is given in Fig.9a. The simulation result using current controller is given in Fig.9b.

2.An intelligent motion planning and navigation system for omnidirectional mobile robot using fuzzy logic presented by Zavlangas et al. [28]. The fuzzy-rule-base of the proposed system combines the repelling effect, which is related to the distance and the angle between the robot and nearby obstacles, with the attracting effect produced by the distance and the angular difference between the actual direction and position of the robot and the final configuration, to generate actuating commands for the mobile platform. The simulation result for the above model is given in Fig. 10a. The simulation result using current controller is given in Fig. 10b.

\section{Experimental Results}

To show the effectiveness of the proposed control system and authenticity of the technique, a variety of real time experiments were conducted using Khepra-III mobile robot (Fig. 11). The mobile robot has 10 infrared sensors and 5 ultrasonic sensors mounted around the front periphery of the mobile robot in order to sense the front, left, right obstacle distance from robot and target angle to 


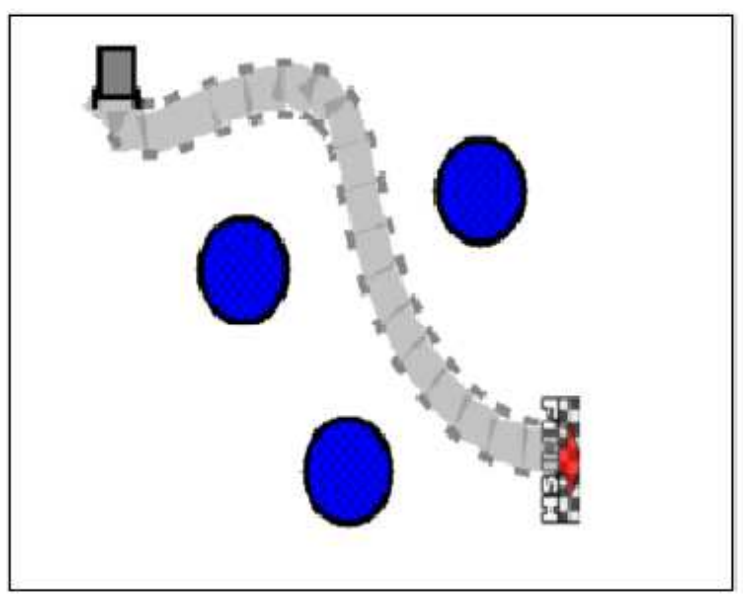

(a) Path obtained by Engedy et al. [27]

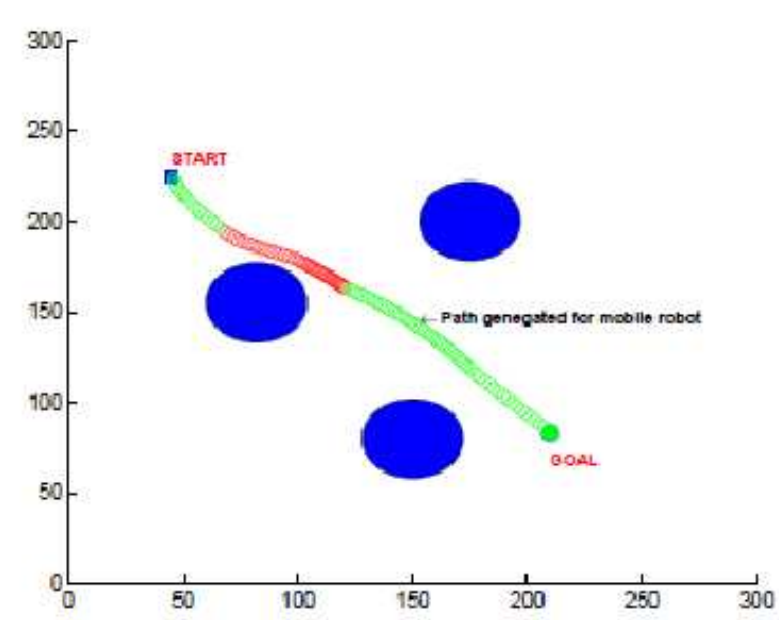

(b) Path obtained by MANFIS controller

Fig. 9

the goal. Two different cases in Fig. 7 and Fig. 8, which are already verify in the simulation link, have been verify experimentally in Fig. 12(a-g) and Fig. 13(a-i) to show the robustness of the designed MANFIS controller. It is shown that the motion planning of mobile robot with proposed hybrid technique has better performance than the fuzzy as well as neural controller in terms of positioning accuracy and obstacle avoidance. Table 2 shows the path length cover by the robot in simulation and in the experimental tests scenario during target searching. The path length traced by the experimentally nearly same as the path traced by the mobile robot during simulation link.

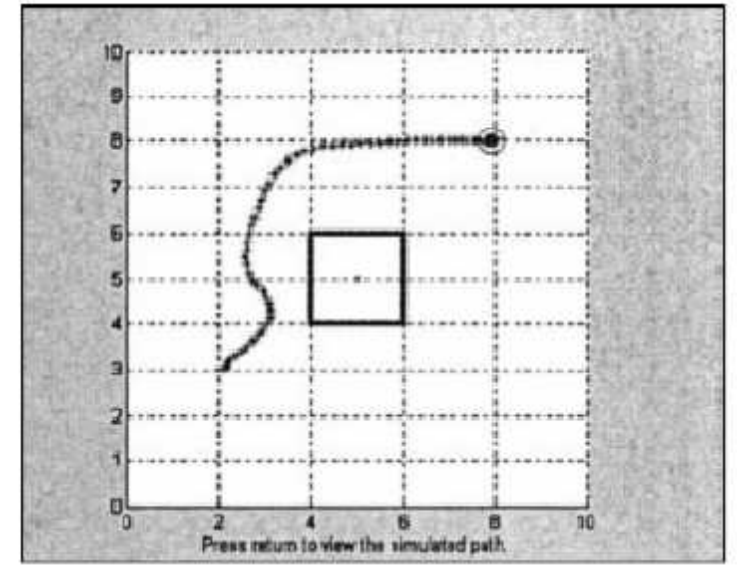

(a) Path obtained by Zavlangas et al. [28]

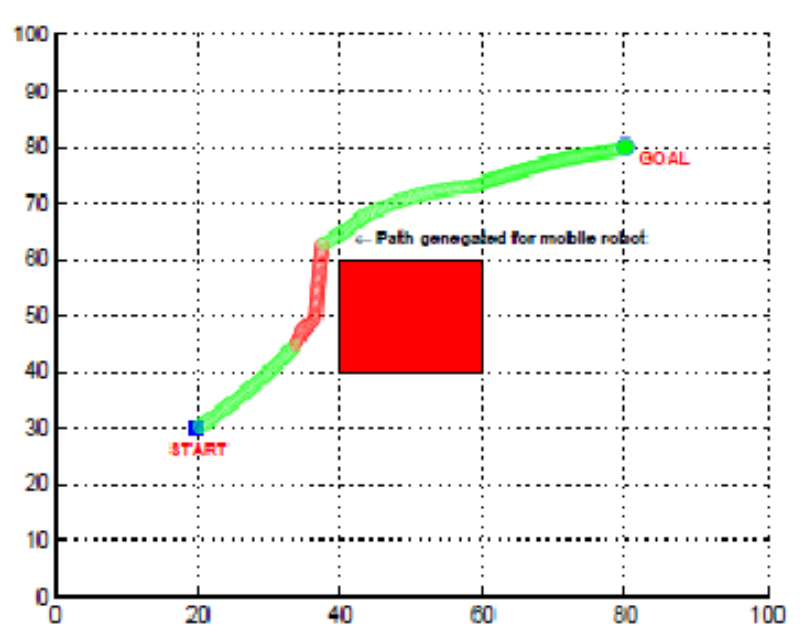

(b) Path obtained by MANFIS controller

Fig. 10

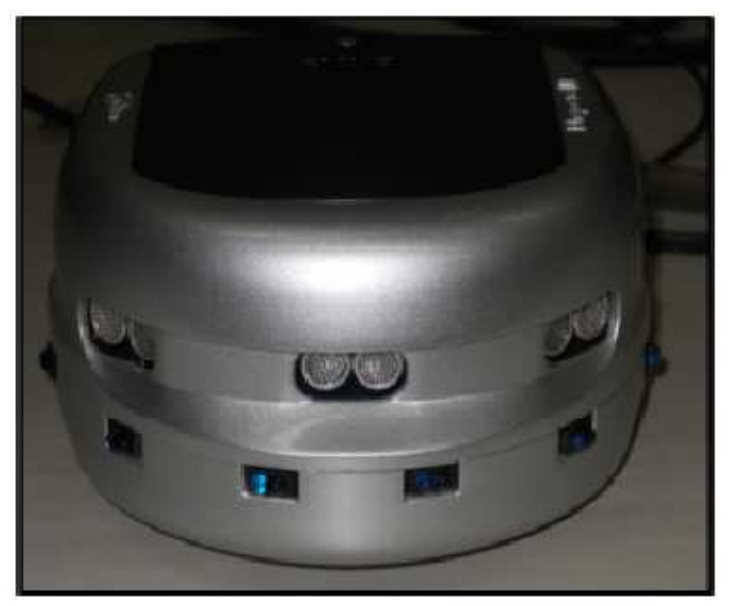

Fig. 11: Khepra-III Mobile Robot 

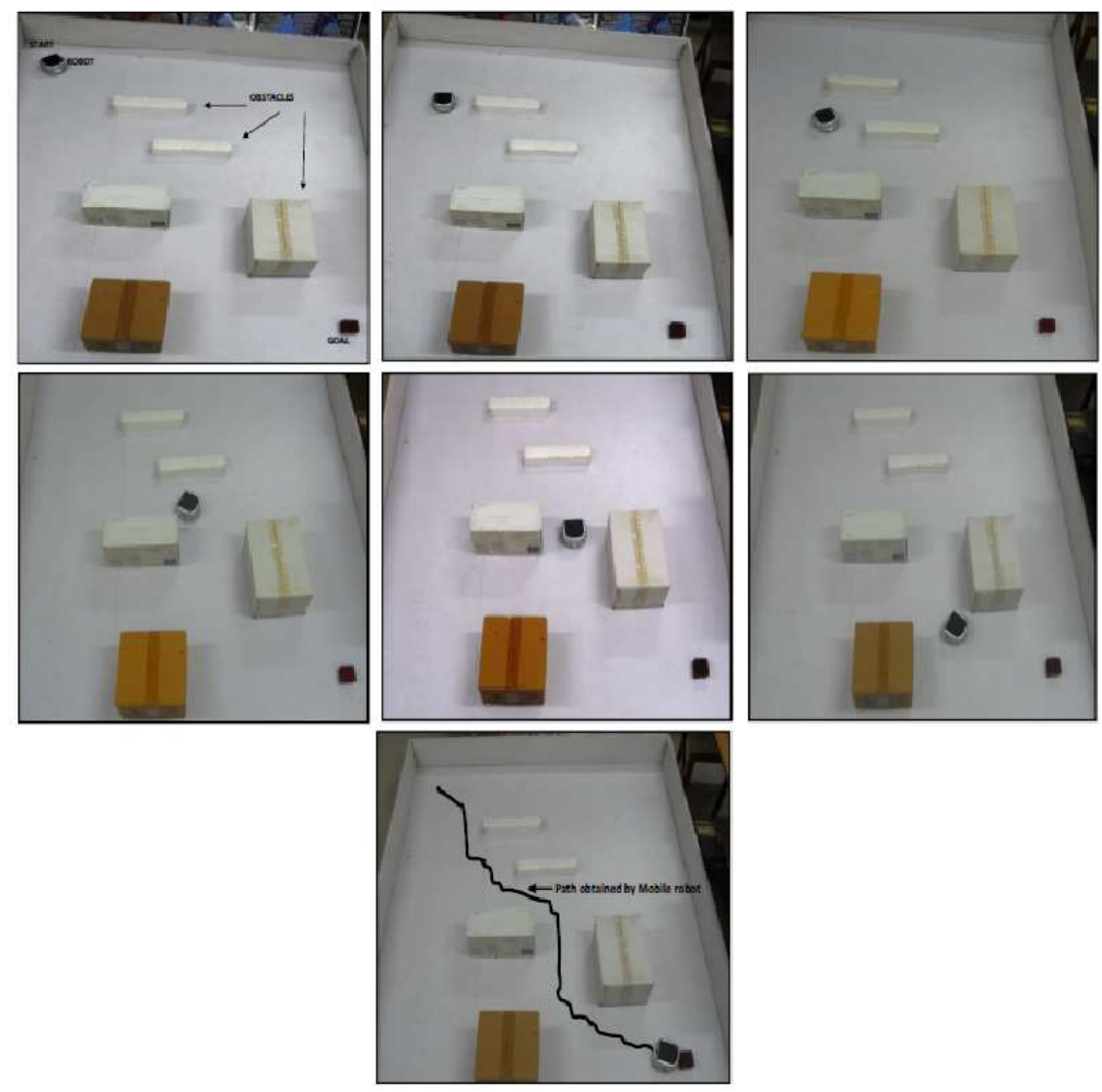

Fig. 12: Experimental set up for navigation of mobile robot in the similar environment shown in Fig. 7

Table 1: Khepra-III Mobile Robot Specifications

\begin{tabular}{|r|l|}
\hline Processor & DsPIC 30F5011 at 60MHz \\
\hline RAM & $4 \mathrm{~KB}$ on DsPIC \\
\hline Speed Max & $0.5 \mathrm{~m} / \mathrm{s}$ \\
\hline Sensors & $\begin{array}{l}10 \text { Infra-red proximity and } \\
\text { ambient light sensors with up } \\
\text { to 30cm range, 5 Ultrasonic } \\
\text { sensors with range 20cm to 4 } \\
\text { meters. }\end{array}$ \\
\hline Power & $\begin{array}{l}\text { Power Adapter Swapable } \\
\text { Lithium-Polymer battery pack } \\
(1350 \mathrm{mAh}) .\end{array}$ \\
\hline Size & $130 \mathrm{~mm}$ Height \\
\hline Diameter & $70 \mathrm{~mm}$ \\
\hline Weight & Approx. 690 g \\
\hline Payload & Approx. 2000 g \\
\hline
\end{tabular}

\section{Concluding Remarks}

This paper has discussed the adaptive navigation for a mobile robot using multiple adaptive neuro-fuzzy inference system (MANFIS) in a completely or partially unknown environment. Adaptive neuro-fuzzy inference
Table 2: Path length cover by robot in simulation and experiment to reach target

\begin{tabular}{|l|l|l|l|}
\hline No. & $\begin{array}{l}\text { Path length in } \\
\text { simulation }\end{array}$ & $\begin{array}{l}\text { Path length in real } \\
\text { time experiment }\end{array}$ & \% of error \\
\hline Scenario-1 & $1.74 \mathrm{~m}$ (Fig. 7) & $1.77 \mathrm{~m}($ Fig. 12(g)) & 2.2 \\
\hline Scenario-2 & $2.06 \mathrm{~m}$ (Fig. 8) & $2.19 \mathrm{~m}($ Fig. 13(i)) & 5.9 \\
\hline
\end{tabular}

system (ANFIS) can be implemented to learn FIS and to identify and refine the various parameters in membership functions and fuzzy rules using training dataset values. The current adaptive navigational controller learns and develops the required information for mobile robot to reach at desired goal. Different simulation results were performed to show the capability of the motion controller. It was found that the proposed adaptive fuzzy controller can be adaptable to any kind of complex environments. Finally the simulation results were compared with real time experimental results to prove the authenticity of the proposed algorithm. Future work can be extended for a single mobile robot navigating in dynamic environment. It will be more interesting if we can be used multiple mobile robots instead of a single mobile robot. 

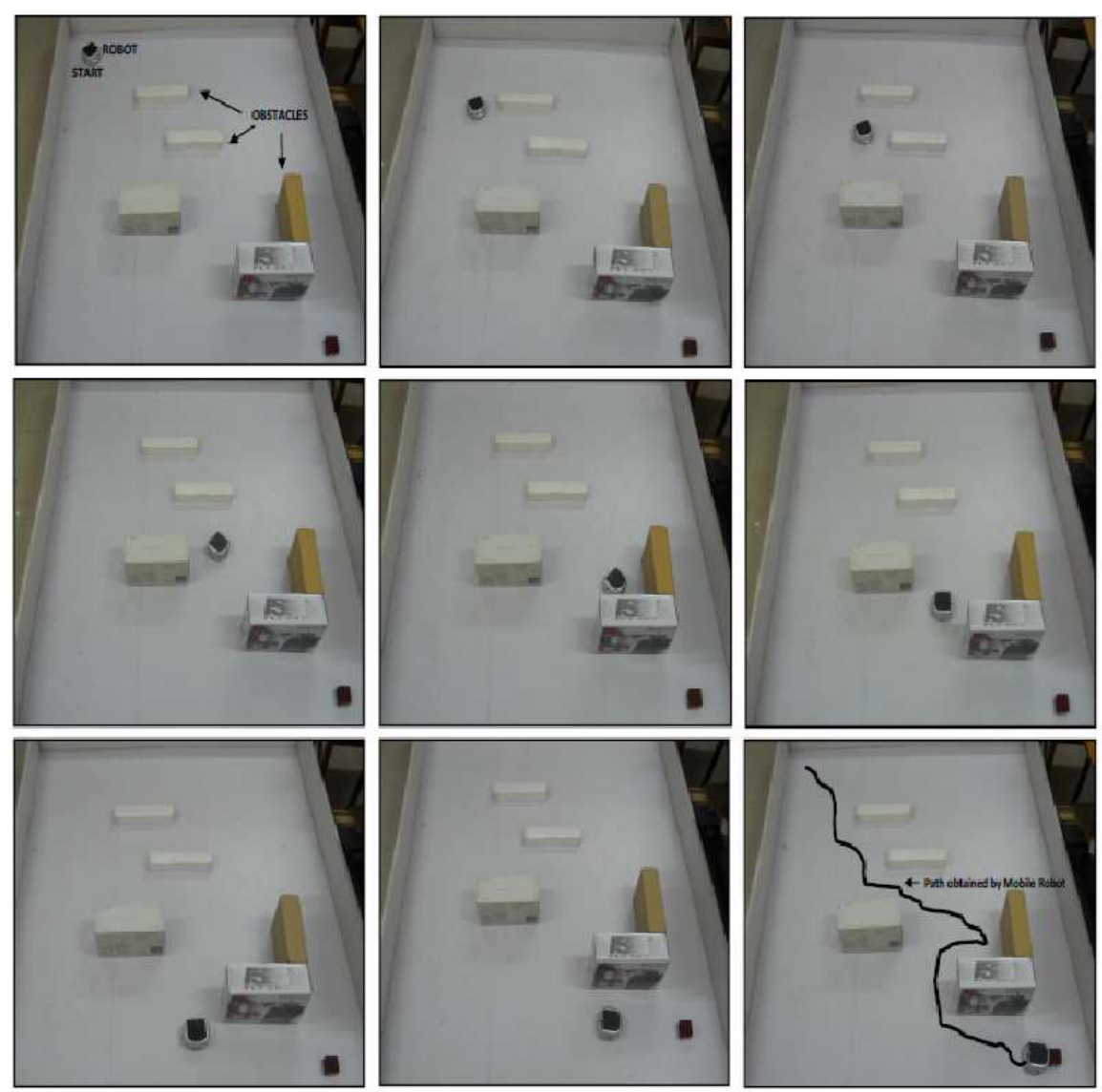

Fig. 13: Experimental set up for navigation of mobile robot in the similar environment shown in Fig. 8

\section{References}

[1] J. C. Latombe, Robot Motion Planning, Kluwer Academic Publishers, New York, (1990).

[2] J.E. Canny, The Complexity of Robot Motion Planning, Cambridge, MA: MIT Press, (1988).

[3] T. L. Perez, A simple motion planning algorithm for general robot manipulators, IEEE Journal of Robotics and Automation, 3, 224-238, (1987).

[4] D. Leven, M. Sharir, Planning a purely translational motion for a convex object in two dimensional space using generalized voronoi diagrams, Discrete \& Computational Geometry, 2, 9-31 (1987).

[5] M. Weigl, B. Siemiaatkowska, K. A. Sikorski, A. Borkowski, Grid-based mapping for autonomous mobile robot, Robotics and Autonomous Systems, 11, 13-21 (1993).

[6] W. Regli, Robot Lab: Robot Path Planning, Lectures Notes of Department of computer Science, Drexel University, (2007).

[7] O. Khatib, Real time Obstacle Avoidance for manipulators and Mobile Robots, In Proceedings IEEE International Conference on Robotics and Automation, March 25-28, Missouri, 500-505 (1985).

[8] K. Fujimura, Path planning with multiple objectives, Robotics \& Automation Magazine, IEEE, 3, 33-38 (1996).
[9] M. Y. Ibrahim, Study on Mobile Robot Navigation Techniques, IEEE International Conference on Industrial Technology, December 8-10, Tunisia, 1, 230-236 (2004).

[10] R. Huq, G.K.I. Mann, R.G. Gosine, Mobile robot navigation using motor schema and fuzzy content behavior modulation, Applied soft computing, 8, $422-436$ (2008).

[11] M.F. Selekwa, D.D. Dunlap, D. Shi, E.G. CollinsJr., Robot navigation in very cluttered environment by preference based fuzzy behaviors, Robotics and Autonomous Systems, 56, 231-246 (2008).

[12] F. Abdessemed, K. Benmahammed, E. Monacelli, A fuzzy based reactive controller for a non-holonomic mobile robot, Robotics and Autonomous Systems, 47, 31-46 (2004).

[13] S.K. Pradhan, D.R. Parhi, A.K. Panda, Fuzzy logic techniques for navigation of several mobile robots, Applied soft computing, 9, 290-304 (2009).

[14] O. Motlagh, S.H. Tang, N. Ismail, Development of a new minimum avoidance system for behavior based mobile robot, Fuzzy Sets and Systems, 160, 1929-1946 (2009).

[15] J. Velagic, N. Osmic, B. Lacevic, Neural Network Controller for Mobile Robot Motion Control, International Journal of Electrical and Computer Engineering, 3, 427-432 (2008).

[16] M.K. Singh, D.R. Parhi, Intelligent Neuro-Controller for Navigation of Mobile Robot, Proceedings of the International 
Conference on Advances in Computing, Communication and Control, 23-24 January, Mumbai, Maharashtra, India, 123128 (2009).

[17] V. Castro, J.P. Neira, C.L. Rueda, J.C. Villamizar, L. Angel, Autonomous Navigation Strategies for Mobile Robots using a Probabilistic Neural Network (PNN), 33rd Annual Conference of the IEEE Industrial Electronics Society (IECON), 5-8 November, Taipei, Taiwan, 2795-2800 (2007).

[18] S.X. Yang, M. Meng, An efficient neural network approach to dynamic robot motion planning, Neural Networks, 13, 143148 (2000).

[19] J.S.R. Jang, 1993, ANFIS: Adaptive network-based fuzzy inference system. IEEE Transaction on System, Man and Cybernetics -part B, 23, 665-685 (1993).

[20] K.T. Song, L.H. Sheen, Heuristic fuzzy-neuro network and its application to reactive navigation of a mobile robot, Fuzzy Sets and Systems, 110, 331-340 (2000).

[21] W. Li, C. Ma, F.M. Wahl, A neuro-fuzzy system architecture for behavior based control of a mobile robot in unknown environments, Fuzzy Sets and Systems, 87, 133-140 (1997).

[22] S.K. Pradhan, D.R. Parhi, A.K. Panda, Neuro-fuzzy technique for navigation of multiple mobile robots, Fuzzy Optimum and Decision Making, 5, 255-288 (2006).

[23] S. Nefti, M. Oussalah, K. Djouani, J. Pontnau, Intelligent Adaptive Mobile Robot Navigation, Journal of Intelligent and Robotic Systems, 30, 311-329 (2001).

[24] Kim.C. Ng, Mohan.M. Trivedi, A Neuro-Fuzzy Controller for Mobile Robot Navigation and Multi robot Convoying, IEEE Transactions on systems, man, and cybernetics-part B: Cybernetics, 28, 829-840 (1998).

[25] N.B. Hui, V. Mahendar, D.K. Pratihar, Time-optimal collision-free navigation of a car-like mobile robot using neuro-fuzzy approaches, Fuzzy Sets and Systems, 157, 21712204 (2006).

[26] J. Godjevac, N. Steele, Neuro-fuzzy control of a mobile robot, Neuro-computing, 28, 127-143 (1999).

[27] I. Engedy, G. Horvth, Artificial Neural Network based Local Motion Planning of a Wheeled Mobile Robot, CINTI 2010, 11th IEEE International Symposium on Computational Intelligence and Informatics, 1820 November, Budapest, Hungary, (2010).

[28] G.Z. Panagiotis, S.G. Tzafestas, Motion Control for mobile robot obstacle avoidance and navigation: A fuzzy logic-based approach. Journal of systems analysis modeling simulation 43, 1625-1637 (2003).

[29] The Math Works Company, Natick, MA, Fuzzy Toolbox Users Guide of MATLAB.

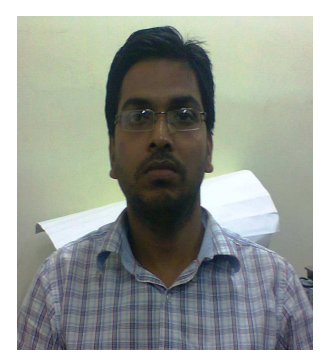

Prases K. Mohanty is presently working as a Senior Research Fellow in National Institute of Technology, Rourkela, Odisha, India. $\mathrm{He}$ is currently pursuing $\mathrm{PhD}$ under same institute. His research areas includes Mobile robot path planning using soft computing

techniques.

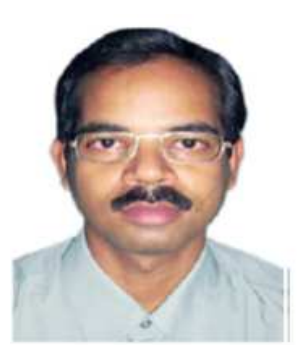

Dayal R. Parhi received his first Ph.D. in Mobile Robotics from Cardiff School of Engineering, UK and second Ph.D. in Vibration Analysis of Cracked structures from Sambalpur University. He has 20 years of research and teaching experience. Presently, he is engaged in Mobile robot Navigation research, and a faculty member in Department of Mechanical Engineering, National Institute of Technology Rourkela. 